\title{
Analysis and modeling for random telegraph noise of GIDL current in saddle MOSFET for DRAM application
}

\author{
Dyukyoung Moon ${ }^{1}$, Hyunseul Lee ${ }^{1}$, Changhwan Shin ${ }^{2 b)}$, \\ and Hyungcheol Shin ${ }^{1 \mathrm{a})}$ \\ ${ }^{1}$ Department of Electrical and Computer Engineering, Seoul National University, \\ 1 Gwanak-ro, Gwanak-gu, Seoul 151-744, Republic of Korea \\ ${ }^{2}$ School of Electrical and Computer Engineering, University of Seoul, \\ 163 Seoulsiripdae-ro, Dongdaemun-Gu, Seoul 130-743, Republic of Korea \\ a)hcshin@snu.ac.kr \\ b)cshin@uos.ac.kr
}

Abstract: The characteristics of the Random Telegraph Noise (RTN) in Gate-Induced Drain Leakage (GIDL) current are first investigated. Based on the Hurkx model, a new model is developed for the TrapAssisted-Tunneling (TAT) and Band-to-Band Tunneling (BBT) regimes in Saddle Metal-Oxide-Semiconductor Field-Effect Transistor (Saddle MOSFET) for DRAM applications. The three-dimensional Technology-based Computer Aided Design (TCAD) simulator is used to quantitatively analyze and model the RTN. The RTN amplitude in GIDL current (i.e., $\Delta \mathrm{I} / \mathrm{I}$ ) increases with increasing drain-to-gate voltage $\left(V_{D G}\right)$ in the TAT regime, whereas $\Delta \mathrm{I} / \mathrm{I}$ decreases with increasing $V_{D G}$ in the BBT regime. Simulation results are well matched to the results estimated by the newly-proposed equations derived from the Hurkx model. This model would open a new pathway for the RTN analysis in DRAM devices.

Keywords: gate-induced drain leakage (GIDL), random telegraph noise (RTN), trap-assisted-tunneling (TAT), band-to-band tunneling (BBT)

Classification: Electron devices, circuits, and systems

\section{References}

[1] Y. Mori, H. Yoshimoto, K. Takeda and Y. Ren-ichi: J. Appl. Phys. 111 (2012) 104513. DOI:10.1063/1.4721658

[2] M. Rosar, B. Leroy and G. Schweeger: IEEE Trans. Electron Devices 47 (2000) 154. DOI:10.1109/16.817581

[3] J. Yu and K. Aflatooni: IEEE Univ./Govern./Industry Microelectronics Symposium16th Biennial (2006) 191.

[4] A. Alnuaimi, A. Nayfeh and V. Koldyaev: J. Appl. Phys. 113 (2012) 044513. 
DOI:10.1063/1.4789382

[5] B. Oh, H.-J. Cho, H. Kim, Y. Son, T. Kang, S. Park, S. Jang, J.-H. Lee and H. Shin: IEEE Trans. Electron Devices 58 (2011) 1741. DOI:10.1109/TED. 2011.2126046

[6] G. A. M. Hurkx, D. B. M. Klaassen and M. P. G. Knuvers: IEEE Trans. Electron Devices 39 (1992) 331. DOI:10.1109/16.121690

[7] G. A. M. Hurkx, H. C. de Graaff, W. J. Kloosterman and M. P. G. Knuvers: IEEE Trans. Electron Devices 39 (1992) 2090. DOI:10.1109/16.155882

[8] G. A. M. Hurkx: Solid-State Electron. 32 (1989) 665. DOI:10.1016/00381101(89)90146-9

[9] K.-H. Park, K.-R. Han and J.-H. Lee: IEEE Electron Device Lett. 26 (2005) 690. DOI:10.1109/LED.2005.854381

[10] K.-H. Park, K.-R. Han, Y. M. Kim and J.-H. Lee: IEEE Electron Device Lett. 27 (2006) 759. DOI:10.1109/LED.2006.880833

[11] Sentaurus Inc.: TCAD Manual, 9-2011.

[12] H. Kim, K. Kim, T.-K. Oh, S.-Y. Cha, S.-J. Hong, S.-W. Park and H. Shin: IEDM (2009) 271. DOI:10.1109/IEDM.2009.5424370

\section{Introduction}

In the state-of-the-art transistors for Dynamic Random Access Memory (DRAM), non-negligible random variations in device characteristics have been emerged. One of the random variations is Random Telegraph Noise (RTN) originated from the capture and emission processes of carriers into the traps in an oxide layer of the device [1]. As of now, this would significantly degrade the retention time in DRAM device.

Off-state leakage in MOSFET is one of the major issues for retention time degradation in DRAM [2]. As the DRAM device dimensions keep shrinking down, it becomes more susceptible to the off-state leakage current, especially the GIDL current [3]. This GIDL current, which is primarily associated with the trap-assisted-tunneling (TAT) in low $V_{D G}$ regime and the band-to-band tunneling (BBT) in high $V_{D G}$ regime, is a significant leakage component in modern DRAM devices [4].

However, most of the previous works on the characterization of RTN has been limited to the RTN in channel or gate leakage current of DRAM devices. The RTN amplitude in GIDL current have been studied but only in the BBT regime [5]. Because of the smaller applied voltage in current DRAM devices, the TAT mechanism would be more dominant in the GIDL current of the DRAM devices. Therefore, a precise understanding of the TAT mechanism related to the RTN is urgently necessary for today's DRAM device design consideration.

In this work, the RTN amplitude in the GIDL current in the TAT region is derived by the Hurkx model $[6,7,8]$. Then, the RTN amplitude in the GIDL current estimated by the proposed model is compared against that obtained from the TCAD simulation data in Saddle Metal-Oxide-Semiconductor FieldEffect Transistor (Saddle MOSFET), as in [9, 10]. 


\section{Nominal saddle MOSFET design and simulation for RTN}

Fig. 1 shows the three-dimensional (3-D) bird's-eye view of a Saddle MOSFET. The nominal device parameters for the Saddle MOSFET are as follows: the physical channel length $\left(L_{G}\right)=20 \mathrm{~nm}$, the junction depth of lightly-doped drain region $\left(\mathrm{LDD} \mathrm{X}_{\mathrm{j}}\right)=31 \mathrm{~nm}$, the recess depth $=60 \mathrm{~nm}$, and the gate oxide thickness $=3.5 \mathrm{~nm}$. The channel/body region is uniformly doped with $10^{17} \mathrm{~cm}^{-3}$, the source/drain region is doped following Gaussian distribution (i.e., the peak concentration on the surface of the source/drain $=$ $3 \times 10^{20} \mathrm{~cm}^{-3}$ and that of the LDD source/drain region $\left.=1 \times 10^{19} \mathrm{~cm}^{-3}\right)$. The source/drain's overlap length to the side-gate is $0 \mathrm{~nm}$, the Si-substrate overlap length to the side-gate $\left(\mathrm{L}_{\text {ov_side }}\right)$ is $10 \mathrm{~nm}$, and the gate width is $20 \mathrm{~nm}$.

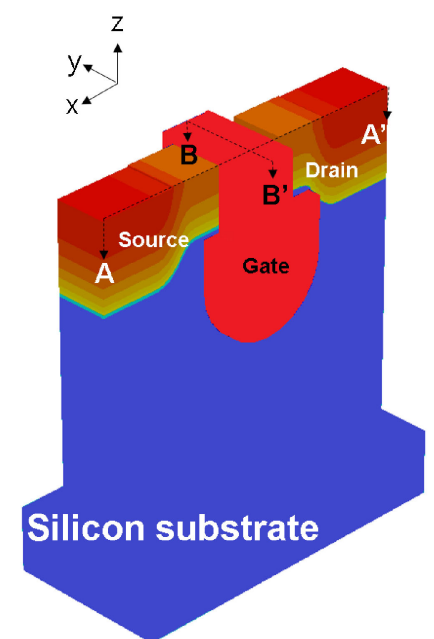

(a)

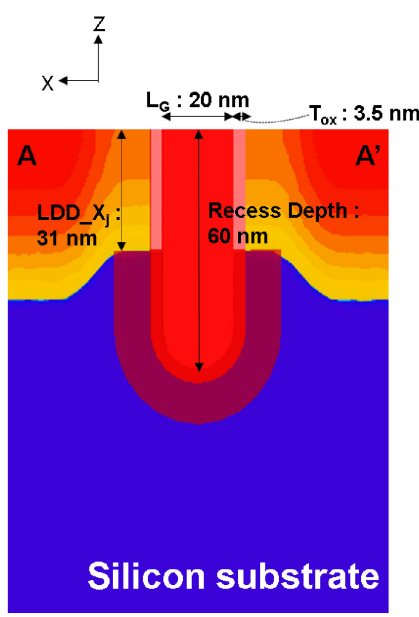

(b)

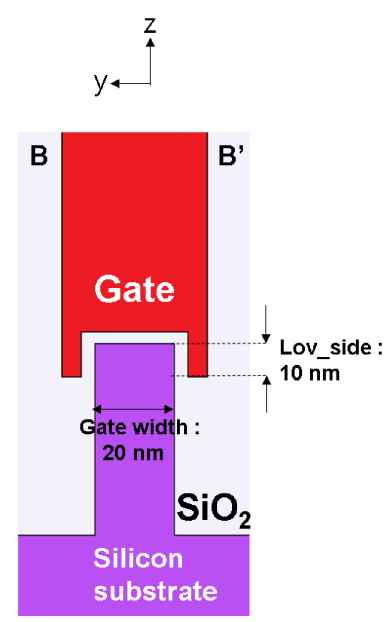

(c)

Fig. 1. (a) 3-D bird's-eye view of Saddle MOSFET, and (b) cross-sectional view along the channel $\mathrm{A}-\mathrm{A}^{\prime}$ and (c) across the channel B-B'.

To run the TCAD simulation for the Saddle MOSFET, following models are included and activated: band gap narrowing model, Shockley-Read-Hall (SRH) recombination model, Hurkx TAT \& BBT model, doping dependent mobility degradation model, high-field saturation mobility model in which carrier drift velocity is no longer proportional to the electric field in high field region, and electron's normal mobility model which is used as mobility degradation at interfaces [11]. In addition, the Fermi Dirac statistics [11] are applied to all the simulations. Temperature is set to $300 \mathrm{~K}$, unless otherwise specified, interface trap density is set to $1 \times 10^{13} \mathrm{~cm}^{-2}$, to see the TAT mechanism in great detail. Using the models mentioned above, the GIDL current difference is investigated, when an electron is trapped or de-trapped in the oxide trap site. The oxide trap is assumed to an acceptor-like trap [i.e., it is neutral (negatively charged) when the trap is empty (filled with an electron)], the position of which is specified in Fig. 2. 


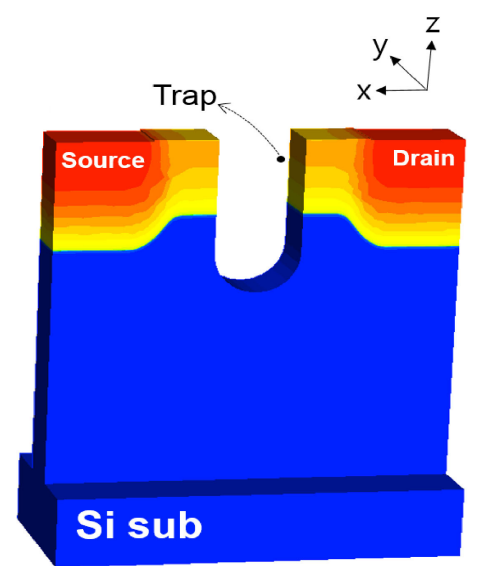

(a)

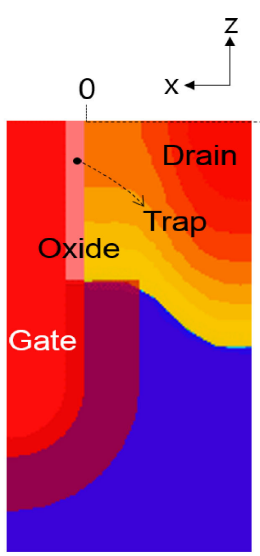

(b)

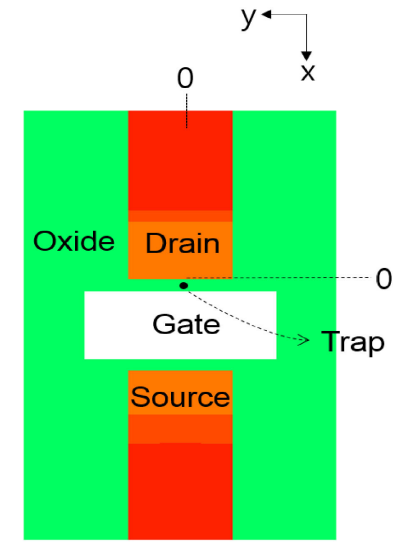

(c)

Fig. 2. Cross-sectional views in (a) $x-y-z$ plane, (b) x-z plane and (c) $x-y$ plane to show the physical location of an oxide trap. Note that $\left(\mathrm{x}_{\mathrm{T}}, \mathrm{y}_{\mathrm{T}}, \mathrm{z}_{\mathrm{T}}\right)=(0.1 \mathrm{~nm}, 0 \mathrm{~nm}$, $-10 \mathrm{~nm})$. The position of the origin $\mathrm{x}=0$ is at the $\mathrm{Si} / \mathrm{SiO}_{2}$ interface at the drain, $\mathrm{y}=0$ is at the center of the gate width, and $z=0$ is the top of the Saddle structure.

\section{RTN modeling}

\subsection{Trap-Assisted-Tunneling (TAT) regime}

When the drain-to-gate bias $\left(V_{D G}\right)$ is small, the RTN amplitude in GIDL current can be derived from the Hurkx model as follows [12]:

$$
\begin{gathered}
I \approx A(1+\Gamma) \\
A=q \frac{n_{i}}{\tau} \exp \left(-\frac{\left|E_{i}-E_{t}\right|}{k_{B} T}\right)
\end{gathered}
$$

where $q$ is $1.6 \times 10^{-19} \mathrm{C}, n_{i}$ is the intrinsic carrier concentration, $\tau$ is the lifetime of carriers, $E_{i}$ is the intrinsic Fermi level, $E_{t}$ is the trap energy level, $k_{B}$ is the Boltzmann constant, and $\mathrm{T}$ is temperature in Kelvin. The function $\Gamma$, which accounts for the effects of the trap-assisted-tunneling on both the density of carriers captured by a single trap and the emission rate of carriers out of the single trap, is given by:

$$
\begin{gathered}
\Gamma(F)=2 \sqrt{3 \pi}\left(\frac{F}{F_{\Gamma, n}}\right) \exp \left[\left(\frac{F}{F_{\Gamma, n}}\right)^{2}\right] \\
F_{\Gamma, n}=\frac{\sqrt{24 m_{n}^{*}\left(k_{B} T\right)^{3}}}{q \hbar},
\end{gathered}
$$

where $F$ is the surface electric field at the drain-to-gate overlap region, $\mathrm{m}_{\mathrm{n}}{ }^{*}$ is the effective mass of carriers, and $\hbar$ is Plank's constant divided by $2 \pi . F_{\Gamma, n}$ includes a term for temperature which means that the TAT mechanism is dependent on temperature. The current fluctuation by RTN is described as follows:

$$
\frac{\Delta I}{I}=\frac{\Gamma(F+\Delta F)-\Gamma(F)}{1+\Gamma(F)}
$$


where $\Delta F$ indicates the fluctuation of the electric field by an electron captured at the trap site. The RTN in the GIDL current can be derived using Eq. (1)-(5), and is represented in the Eq. (6) below.

$$
\frac{\Delta I}{I}=\left(1+\frac{\Delta F}{F}\right) \exp \left(\frac{2 F \Delta F}{F_{\Gamma, n}^{2}}\right)-1
$$

\subsection{Band-to-Band Tunneling regime}

The GIDL current can be expressed as in Eq. (7):

$$
I=S \frac{A}{B} F^{\sigma} \exp \left(-\frac{B}{F}\right)
$$

The parameters in (7) are given by the Hurkx BBT model. $S$ is the crosssectional area, $A=4 \times 10^{14}\left[\mathrm{~cm}^{-3} \cdot \mathrm{s}^{-1}\right], B=1.9 \times 10^{7}[\mathrm{~V} / \mathrm{cm}], \sigma=2.5$, as in [11]. Assuming that $\Delta F$ is sufficiently smaller than $F$, binominal expansion can be applied to the Eq. (7). Then, the RTN amplitude in the GIDL current can be modeled as in the Eq. (8) below:

$$
\frac{\Delta I}{I}=\exp \left(\frac{B}{F^{2}} \Delta F\right)-1
$$

\subsection{Transition regime from TAT to BBT}

In the $V_{D G}$ range of $1.0 \mathrm{~V} \sim 1.6 \mathrm{~V}$, the GIDL current has two mechanisms (TAT and BBT). So in this regime, we need both the Eq. (6) and the Eq. (8):

$$
\frac{\Delta I}{I}=\left(1+\frac{\Delta F}{F}\right) \exp \left(\frac{2 F \Delta F}{F_{\Gamma, n}^{2}}\right)+\exp \left(\frac{B}{F^{2}} \Delta F\right)-2
$$

\section{Results and discussion}

Once an electron is captured in the trap site, the GIDL current is increased (Fig. 3a). This is due mainly to the fact that the trap filled with the electron can enhance the electric field onto the semiconductor surface. The higher electric field can cause higher tunneling process to be occurred, so that the GIDL current is increased with the trap being filled with an electron. On the other hand, the GIDL current is decreased when the trap is empty (not filled with an electron).

We can observe in the condition of empty trap that the TAT mechanism is dominant if $V_{D G}<1.66 \mathrm{~V}$ and the BBT mechanism is dominant if $V_{D G}>$ $1.66 \mathrm{~V}$ [Fig. 3(b)]. At the high $V_{D G}$ region, the BBT is the dominant mechanism for the GIDL current, because the BBT can be strongly happened (1) with the presence of a sufficiently-high electric field and (2) with the band bending larger than energy band gap $\left(E_{g}\right)$. As a result of the higher electric field or voltage used for the BBT regime, a deep-depletion region is also formed underneath the gate-to-drain overlap region. On the other hand, at the low $V_{D G}$ region, electron and hole emissions are carried out via thermal excitation. The thermal excitation becomes more prominent at the low $V_{D G}$ region, whereas the band-to-band tunneling is relatively weak. Lastly, with the power 

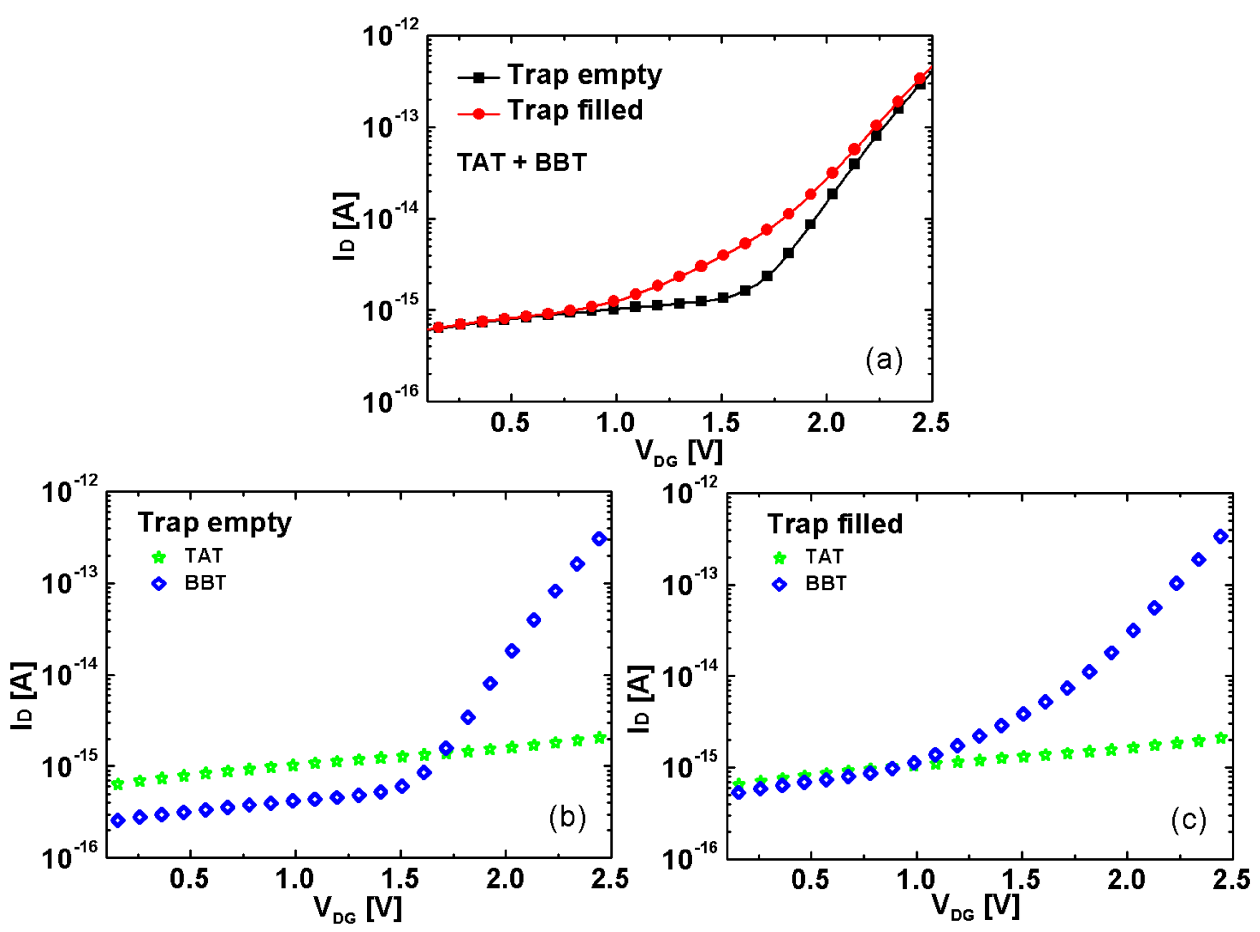

Fig. 3. (a) GIDL current versus $V_{D G}$. Note that the GIDL current is increased with the trap filled with an electron. (b) GIDL current decomposed into the TAT-induced and the BBT-induced GIDL current, when the trap is empty. (c) GIDL current decomposed into the TAT-induced and the BBT-induced GIDL current, when the trap is filled.

supply voltage of $1.2 \mathrm{~V}$ for DRAM application (as suggested in ITRS 2012), it is found that the TAT mechanism mainly drives the GIDL current [Fig. 3(b)]. It is noteworthy to mention that the words used in this paper 'TAT regime' and 'BBT regime' means that it is in the trap empty condition [Fig. 3(b)].

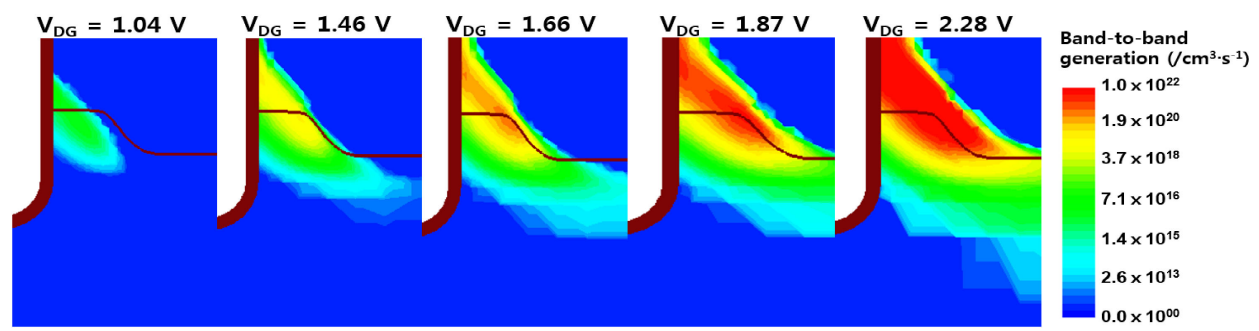

Fig. 4. Contour plots of band-to-band generation rate near and in the drain region, with various $V_{D G}$ values.

Fig. 4 illustrates how the band-to-band generation rate is varied near/in the drain region. The rate becomes higher as $V_{D G}$ increases, which physically indicates that the band-to-band tunneling mechanism is dominant in the GIDL current at higher $V_{D G}$. 


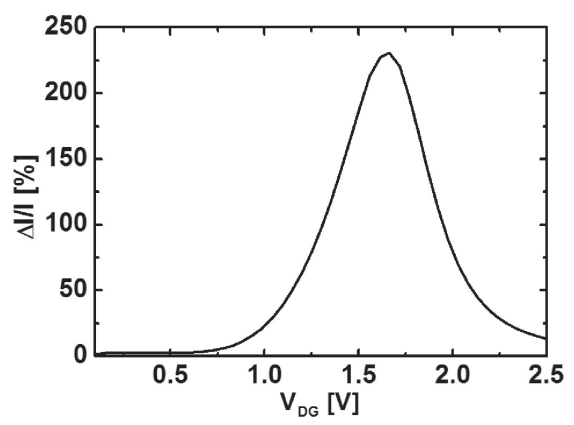

Fig. 5. RTN amplitude in GIDL current (i.e., $\Delta \mathrm{I} / \mathrm{I}$ ) versus $V_{D G}$. Note that the peak of the $\Delta \mathrm{I} / \mathrm{I}$ reaches up to $230 \%$.

Fig. 5 shows the RTN amplitude in the GIDL current (i.e., $\Delta \mathrm{I} / \mathrm{I}$ ) with the trap at the position shown in Fig. 2. The peak value of $\Delta \mathrm{I} / \mathrm{I}$ reaches up to $230 \%$ at $V_{D G}=1.66 \mathrm{~V}$. As $V_{D G}$ increases, the RTN amplitude in the GIDL current correspondingly increases in the TAT regime [which is also expected from the Eq. (6)]. And in the $V_{D G}$ range of $1.0 \mathrm{~V} \sim 1.6 \mathrm{~V}$, it follows the Eq. (9). The $F$ in (6), (9) is almost linearly dependent on $V_{D G}$. If the trap position becomes close to the interface between the oxide and the drain, then the RTN amplitude will be increased. Because the Coulombic force between the trapped charge in the oxide and the interface trap becomes large, chosen is the oxide trap position that has moderate effect to the oxide and drain interface.

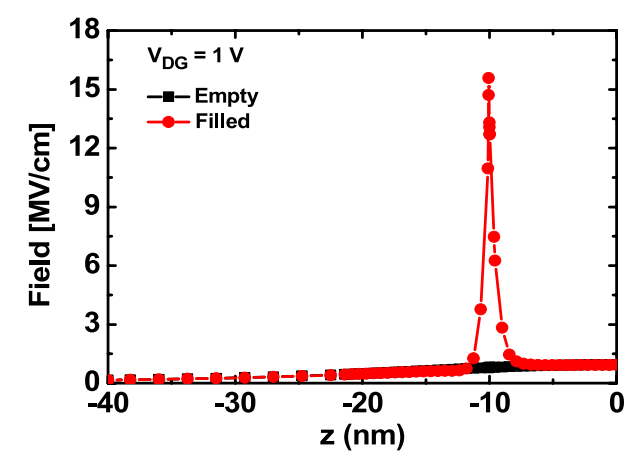

Fig. 6. The electric field of empty trap (black squares) and of filled trap (red circles), along the z-direction which is at $\mathrm{x}=0\left(\mathrm{Si} / \mathrm{SiO}_{2}\right.$ interface $)$ and $\mathrm{y}=0$ (center of the Si fin).

Fig. 6 shows the electric field along the z-direction, for two different situations when $V_{D G}$ of $1 \mathrm{~V}$ is applied. The black squares represents the situation that the trap is empty, and the red circles indicates the other situation that the trap is filled with an electron. The electric field at the trap's position $\left(z_{T}=-10 \mathrm{~nm}\right)$ increases up to $16 \mathrm{MV} / \mathrm{cm}$, when the trap is filled with an electron. For various $V_{D G}$ values, the shape of the electric field distribution along the $z$-direction is almost identical to the one shown in Fig. 6. 
As the interface trap increases, the RTN amplitude decreases like shown in Fig. 7. The effect of the oxide trap to the interface trap is small $(\Delta I)$ compared to the large change of current $(I)$ which is augmented by increasing the interface trap density. So the $\frac{\Delta I}{I}$ is decreased.

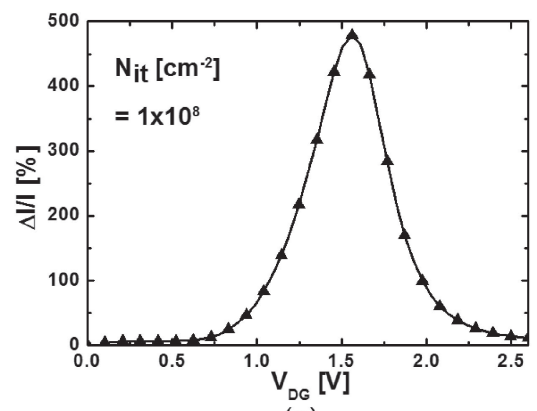

(a)

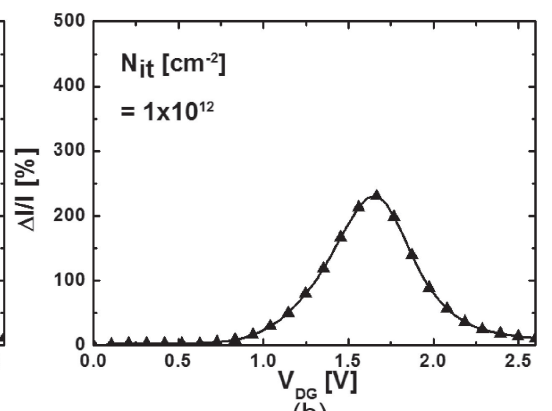

(b)

Fig. 7. The RTN amplitude when the interface trap density is (a) $10^{8} \mathrm{~cm}^{-2}$, (b) $10^{12} \mathrm{~cm}^{-2}$

In the TAT regime, the simulation data are fit to the data estimated from the Eq. (6). But as you can see at the $V_{D G}$ range of $1.0 \sim 1.6 \mathrm{~V}$, the GIDL current is dominantly effected by the TAT mechanism when the situation is in the empty trap condition [Fig. 3(b)]. But in filled trap condition, BBT mechanism is more dominant than TAT mechanism [Fig. 3(c)]. So at that $V_{D G}$ range, we matched the simulation data with the Eq. (9). We substituted $F$ and $\Delta F$ at each point of $V_{D G}$ into the Eq. (6) and Eq. (9) [Fig. 8(a)]. Fig. 8(b) shows $F$ and $\Delta F$, as a function of $V_{D G}$. The calculation results and the simulation results are well matched to each other. There is little difference between them in $1.4 \sim 1.6 \mathrm{~V}$, because the range is corresponding to the transition region from the TAT regime to the BBT regime. As we assumed in deriving the Eq. (6), $\Delta F$ is very small (versus $F$ ). The other fitting parameter $\left(\mathrm{m}_{\mathrm{n}}{ }^{*}\right)$ used in the Eq. (4) is $0.35 \times m_{0}\left(m_{0}\right.$ : free electron's mass in vacuum).

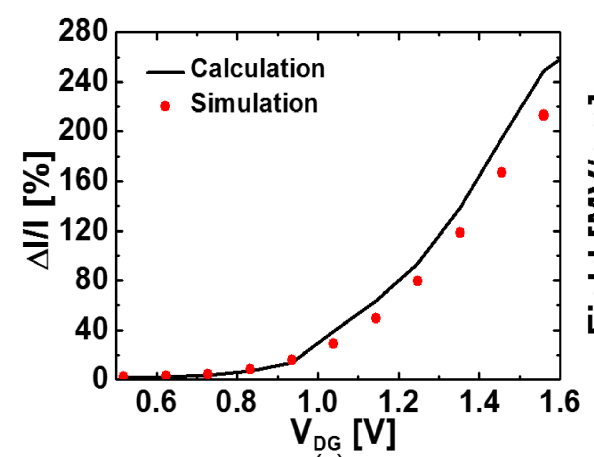

(a)

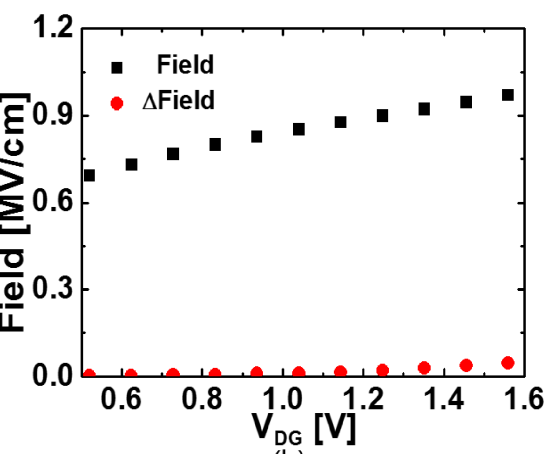

(b)

Fig. 8. (a) Fitting result of RTN amplitude of GIDL current in TAT regime. (b) $F$ and $\Delta F$ of RTN amplitude in GIDL current in TAT regime. 
For the BBT regime, the simulation data are fit to the data estimated from the Eq. (8). We substituted $F$ and $\Delta F$ at each point of $V_{D G}$ into the Eq. (8) [Fig. 9(a)]. Fig. 9(b) shows $F$ and $\Delta F$, as a function of $V_{D G}$. The calculation results and the simulation results are well matched to each other. As the same reason applied for the TAT regime, there is little difference between the simulation result and the calculation result in the range of $V_{D G}=1.6 \sim 1.8 \mathrm{~V}$, because the voltage range is corresponding to the transition region from the TAT regime to the BBT regime. As we assumed in deriving the Eq. (8), $\Delta F$ is very small (versus $F$ ).
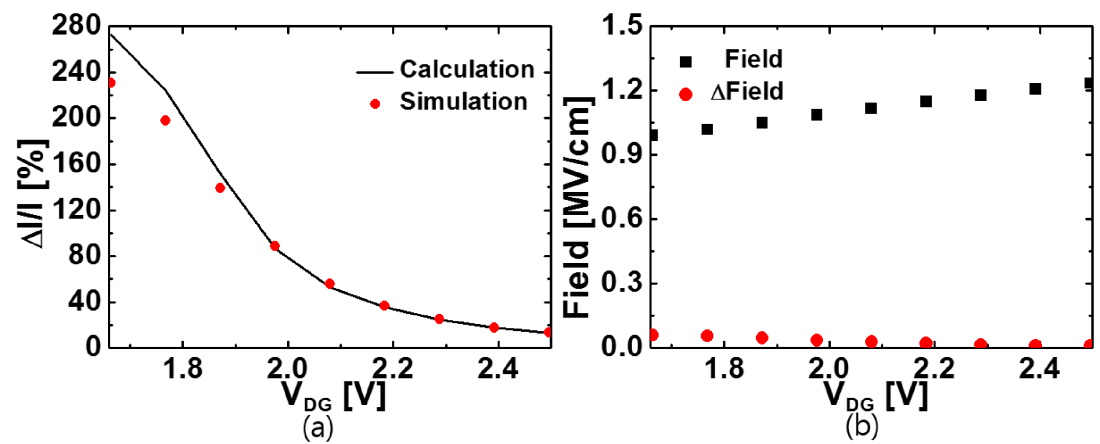

Fig. 9. (a) Fitting result of RTN amplitude of GIDL current in BBT regime. (b) $F$ and $\Delta F$ of RTN amplitude in GIDL current in BBT regime.

\section{Conclusion}

Using three dimensional TCAD simulation, the RTN amplitude of the GIDL current in the Saddle MOSFET for DRAM application has been modeled for two regimes: Trap-Assisted-Tunneling (TAT) and Band-to-Band Tunneling (BBT) regimes. The RTN amplitude in GIDL current which is newly derived from the Hurkx model was excellently agreed to the TCAD simulation data. At power supply voltage of $1.2 \mathrm{~V}$ for DRAM application, the TAT mechanism (vs. BBT mechanism) becomes dominant for GIDL current.

\section{Acknowledgments}

This work was supported by Samsung Electronics co., Ltd., and Inter-university Semiconductor Research Center (ISRC). This work was also supported by Basic Science Research Program through the National Research Foundation of Korea (NRF) funded by the Ministry of Science, ICT and Future Planning (grant number: 2012R1A1A1014011). 